\title{
Efficacy of Mebendazole and Praziquantel against Soil- Transmitted Helminths and Schistosoma mansoni Infections among Schoolchildren in Northwest Ethiopia
}

\author{
Kefale Ejigu, ${ }^{1}$ Tadesse Hailu $\mathbb{D}^{2}{ }^{2}$ and Megbaru Alemu ${ }^{2}$ \\ ${ }^{1}$ Amhara Public Health Institute, Bahir Dar, Ethiopia \\ ${ }^{2}$ Department of Medical Laboratory Science, College of Medicine and Health Sciences, Bahir Dar University, PO Box 79, Ethiopia \\ Correspondence should be addressed to Tadesse Hailu; tadessehailu89@gmail.com
}

Received 15 November 2020; Revised 4 July 2021; Accepted 9 July 2021; Published 16 July 2021

Academic Editor: Francesca Mancianti

Copyright () 2021 Kefale Ejigu et al. This is an open access article distributed under the Creative Commons Attribution License, which permits unrestricted use, distribution, and reproduction in any medium, provided the original work is properly cited.

\begin{abstract}
Background. Soil-transmitted helminths (STHs) and Schistosoma mansoni are the main causes of morbidity among schoolchildren in the tropics. A school-based deworming program was launched to control and eliminate the infection in endemic countries including Ethiopia. Although periodic deworming is conducted in endemic areas, the prevalence of the infection is high in the country. In addition, periodic evaluation of the efficacy of the anthelminthic drug is limited. Objective. This study is aimed at checking the efficacy of mebendazole and praziquantel with the respective STHs and Schistosoma mansoni parasites. Methods. A longitudinal study was conducted from February to March 2018 among 422 schoolchildren. Stool samples were collected at baseline and at 2 and 4 weeks posttreatment and were processed using the Kato-Katz technique. Schoolchildren positive for STHs were treated with mebendazole and those positive for Schistosoma mansoni with praziquantel. After two weeks, a second round of stool was collected and examined, and then, single-dose redosing was given to each positive child. Lastly, the third stool sample was collected two weeks after the initiation of the redosing and checked for STHs and S. mansoni parasites. A close follow-up of students who were treated was done. All the data were entered and analyzed using SPSS version 20 for analysis. Descriptive statistics was used to compute the cure rate and egg reduction rate of mebendazole and praziquantel. Results. Among 422 participants, the prevalence of STHs, hookworm, Ascaris lumbricoides, and S. mansoni was 44.7\%, 35.1\%, 21.1\%, and $13.9 \%$, respectively. The cure rate of mebendazole against A. lumbricoides increased from $60 \%$ in the single dose to $100 \%$ in redosing after two weeks. The cure rate of mebendazole against hookworm also increased from $32.4 \%$ in the single dose to $91.0 \%$ in the redosing. The cure rate of praziquantel against S. mansoni-infected children was $91.5 \%$ in the first round and $100 \%$ in the redosing phase. There was a 98.6-100\% egg reduction rate in the redosing regimen of both drugs. Conclusion. The cure and egg reduction rates of single-dose mebendazole in the treatment of hookworm and A. lumbricoides are lower at week two than at redosing, while cure and egg reduction rates of single-dose praziquantel are satisfactory to treat $S$. mansoni. Therefore, single-dose praziquantel to $S$. mansoni and redosing of single-dose mebendazole to A. lumbricoides and hookworm infections can be used for treatment purposes.
\end{abstract}

\section{Introduction}

Soil-transmitted helminthiasis and schistosomiasis are among the most common parasitic diseases of the neglected tropical diseases (NTDs) worldwide. Soil-transmitted helminths comprise four species of intestinal helminths, $A$. lumbricoides, T. trichiura, and hookworm (Ancylostoma duodenale and Necator americanus) [1,2]. Global estimates indicated that 819 million, 439 million, and 465 million peo- ple are infected with A. lumbricoides, hookworm spp., and $T$. trichiura, respectively. Similarly, more than 200 million people are estimated to be infected with $S$. mansoni (WHO, 2017). In Ethiopia, an estimated 116.3 million people (comprised of 37.6 million school-age children) live in STHs and Schistosoma endemic areas [3].

A. lumbricoides and T. trichiura are transmitted via ingestion of contaminated food and/or soil whereas hookworm infection is acquired via the active penetration of the 
skin by the larvae. S. mansoni is also transmitted through skin penetration of cercaria [4]. School-age children (SAC) and preschool-age children (PSAC) are the most vulnerable population groups both for STHs and S. mansoni since they are playing in soil and water [5].

Heavy STH infection has a great effect on nutritional and cognitive status especially in schoolchildren due to the increased metabolic rate, anorexia, and diarrhea. These helminths also cause decreased intake or a functional increase in the body's nutrient requirement by their interference with absorptive surfaces, physical obstruction of intestinal lumen, production of proteolytic substances, and consumption of nutrients intended for the body [6, 7]. S. mansoni on the other hand can cause a wide range of clinical conditions that range from mild cutaneous and pulmonary manifestations to life-threatening conditions like hepatosplenic inflammation and liver fibrosis $[8,9]$.

The three main measures to control soil-transmitted helminthiasis and schistosomiasis include mass drug administration (MDA); water, sanitation, and hygiene (WASH); and health education. Periodic deworming of the high-risk population ensures that the levels of infection are kept below the intensity that is associated with morbidity. In Ethiopia, mebendazole (single $500 \mathrm{mg} / \mathrm{kg}$ oral dose) and praziquantel (single $40 \mathrm{mg} / \mathrm{kg}$ oral dose) are currently used in deworming programs targeting STHs and S. mansoni, respectively [10]. However, some livestock and human studies indicated reduced efficacy of single-dose anthelminthic therapy [1114]. The reduced efficacy of anthelminthic drugs might be due to repeated reinfection in the endemic areas, highintensity infection, repeated use of the same drug, and single-dose administration of anthelminthic drugs [15].

In the study area, institution-based information showed that the prevalence of STHs and S. mansoni infections is high though MDA is regularly given. Therefore, the aim of this study was to check the efficacy of single-dose mebendazole and praziquantel against the respective STHs and S. mansoni infections at day 14 and efficacy of redosing mebendazole and praziquantel against STHs and S. mansoni infections, respectively, after day 28 .

\section{Materials and Methods}

2.1. Study Design, Area, and Period. A school-based longitudinal study was carried out in Andasa primary school, Bahir Dar Zuria district, Amhara region, northwest Ethiopia, from February to March 2018. The study area is located $560 \mathrm{~km}$ northwest of the country's capital, Addis Ababa. The elevation of the area ranges from 1700 to $2300 \mathrm{~m}$ above sea level. The area receives an average annual rainfall of about $1035 \mathrm{~mm}$. The minimum and maximum temperatures lie at $10^{\circ} \mathrm{C}$ and $32^{\circ} \mathrm{C}$, respectively. There are two rivers (Abay and Andasa), and people frequently encounter water contact while swimming, bathing, and washing clothes and in microirrigations. Deworming has been conducted once a year as part of a control program of STHs and S. mansoni infections (Figure 1).

All schoolchildren in the age range 7-18 years enrolled during the data collection time and their parents who con- sented and provided assent were included in the study. All schoolchildren who had taken any anthelminthic within 3 months prior to data collection were excluded. Participants were selected by systematic random sampling technique, and a total of 422 schoolchildren were included in the study.

2.2. Data Collection. Approximately two grams of stool was collected from each study participant using a clean, dry, and leak-proof container and applicator stick. Stool samples were transported to Amhara Public Health Institute (APHI) for laboratory processing using a cold chain. Kato-Katz thick smears were prepared and examined using a microscope by following the standard operating procedure.

In the Kato-Katz technique, feces were pressed through a mesh screen to remove large particles. A portion of the sieved stool sample was transferred through the hole of the template (41.7 mg of stool) onto a slide. After filling the hole, the template was removed and the remaining sample was covered with a piece of cellophane previously soaked in glycerol that helps to clear the fecal debris around the eggs. The KatoKatz slides were examined with a microscope (10x followed by $40 \mathrm{x}$ magnification).

\subsection{Evaluating the Efficacy of Mebendazole and Praziquantel.} Training on keeping hygiene, shoe wearing, and abstinence from swimming until completing the follow-up was given for those participating schoolchildren in the study. Mebendazole (single $500 \mathrm{mg} / \mathrm{kg}$ oral dose) was provided for the children positive for A. lumbricoides and hookworm parasites based on the baseline results. Praziquantel (single $40 \mathrm{mg} / \mathrm{kg}$ oral dose) was also administered for schoolchildren positive for S. mansoni. After two weeks of initiation, a second round of single stool sample was collected and examined. And then, redosing of mebendazole (single $500 \mathrm{mg} / \mathrm{kg}$ ) and praziquantel (single $40 \mathrm{mg} / \mathrm{kg}$ ) oral dose was provided for those children who were positive in the second round of stool examination. A close follow-up of students to check the adverse drug reaction was done from day one to day 28 . And finally, a third round of single stool sample was collected and examined two weeks after the redosing regimen.

Cure is defined as the absence of $A$. lumbricoides, hookworm, and $S$. mansoni ova or the absence of ova in the stool at day 28 of treatment. "Failure" is defined as the presence of ova of the above parasites at 28 days of treatment initiation:

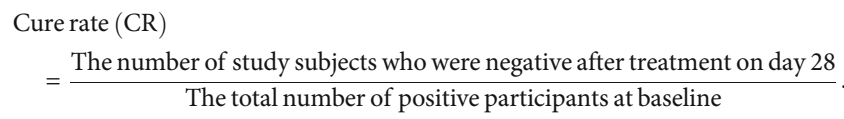

Egg reduction rate (ERR): it was calculated as the ratio of the arithmetic mean of egg count at baseline minus the arithmetic mean of egg count at follow-up divided by arithmetic mean of egg count at baseline and multiplied by 100 . The efficacy of the anthelminthics was interpreted as satisfactory, doubtful, or reduced based on the reference values of the egg reduction rate [15]. 


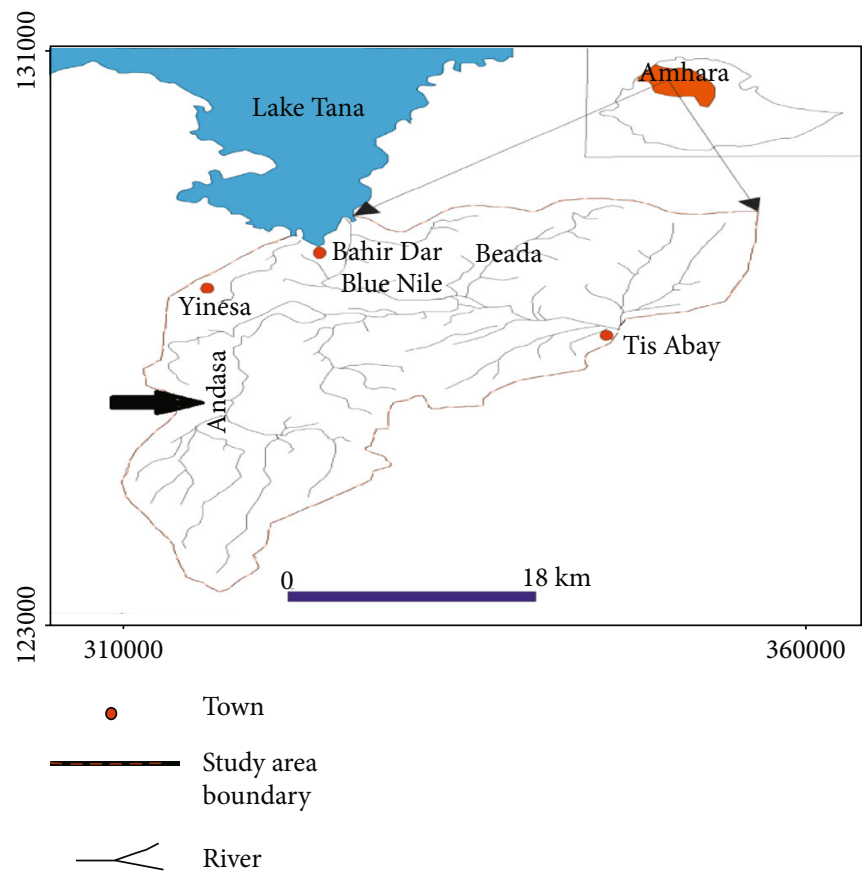

Figure 1: Map of the study area.

2.4. Quality Assurance. The data quality was assured during the preanalytical, analytical, and postanalytical phases. To avoid observer bias, one slide was examined by two laboratory professionals independently. Two Kato-Katz slide smears were examined for a single participant in the second and third rounds of examination, and $15 \%$ of the Kato-Katz slides were randomly selected and checked by the principal investigator.

2.5. Data Entry and Analysis. All the data were entered and analyzed using Statistical Package for the Social Sciences (SPSS) version 20 software. The prevalence of A. lumbricoides, hookworm, and $S$. mansoni was calculated by descriptive statistics. The CR and ERR of mebendazole against $A$. lumbricoides, hookworm, and praziquantel against S. mansoni were computed at day 14 and day 28 of treatment by using the arithmetic mean egg count.

\section{Results}

3.1. Demographic Characteristics of the Study Participants. A total of 422 schoolchildren were recruited in this study. Of the total, the number of female and male participants accounted for $51.9 \%$ and $48.1 \%$, respectively. The mean ( \pm sd) age of the children was $11.87( \pm 1.98)$. The majority $(63.5 \%)$ of the participants were in the $11-14$ years age group (Table 1).

The overall prevalence of helminths and STH infection was $51.2 \%$ and $44.7 \%$, respectively. Children in the $11-14$ age group harboured the majority $(33.6 \%)$ of the helminth infection. More males (28\%) than females (23.2\%) were infected with helminths but not statistically significant (Table 1).
The prevalence of hookworm spp., A. lumbricoides, and S. mansoni was $148(35.1 \%), 89(21.1 \%)$, and $59(13.9 \%)$, respectively (Table 2). Enterobius vermicularis 4 (0.94\%), $H$. nana $2(0.47 \%)$, and Taenia spp $2(0.47 \%)$ were also identified. The prevalence of single, double, and triple infection accounted for $71(32.8 \%), 133(61.6 \%)$, and $12(5.6 \%)$, respectively. Majority of the double infection was due to coinfection of hookworm and A. lumbricoides (22.2\%). Triple infection (hookworm $+A$. lumbricoides $+S$. mansoni) of $5.6 \%$ was also found.

3.2. The Mean Egg Count and Infection Intensity of STHs and Schistosoma mansoni. The mean egg count for individuals with detectable eggs of S. mansoni, A. lumbricoides, and hookworm at the baseline fecal examination was 42, 129, and 281 eggs per gram of feces, respectively (Table 3). All A. lumbricoides and S. mansoni infections were light, and only $5.4 \%$ of hookworm infections had moderate intensity.

3.3. Cure Rate of Mebendazole and Praziquantel. A total of 89 schoolchildren were found to be infected with A. lumbricoides, and 85 of them had taken mebendazole treatment and completed the study. Among 148 hookworm-infected schoolchildren, 145 took mebendazole treatment and completed the follow-up. All 59 S. mansoni-infected schoolchildren took praziquantel and completed the study. We were unable to collect the $2^{\text {nd }}$ stool sample (at day 14) from four A. lumbricoides- and three hookworm-infected schoolchildren since they were absent from school.

The CR of single-dose mebendazole against hookworms and A. lumbricoides was $32.4 \%$ and $59.6 \%$, respectively. Following the redosing regimen, $\mathrm{CR}$ of mebendazole increased to $91.0 \%$ and $100 \%$ to the respective hookworm and A. lumbricoides-infected children. The CR of praziquantel against $S$. 
TABle 1: Demographic characteristics of the study participants with their intestinal helminthic infection status in Andasa primary school, northwest Ethiopia, 2018.

\begin{tabular}{lcccc}
\hline Variable & & Positive, $n(\%)$ & Negative, $n(\%)$ & Total, $n(\%)$ \\
\hline \multirow{2}{*}{ Age } & $7-10$ & $58(13.8 \%)$ & $39(9.2 \%)$ & $97(23 \%)$ \\
& $11-14$ & $142(33.6 \%)$ & $126(29.9 \%)$ & $268(63.5 \%)$ \\
\hline \multirow{2}{*}{ Sex } & $15-18$ & $16(3.8 \%)$ & $41(9.7 \%)$ & $57(13.5 \%)$ \\
\hline Total & Male & $118(28 \%)$ & $85(20.1 \%)$ & $203(48.1 \%)$ \\
\hline
\end{tabular}

TABLE 2: Prevalence of STHs and S. mansoni infections among children at Andasa primary school in Bahir Dar Zuria district, northwest Ethiopia, 2018.

\begin{tabular}{|c|c|c|c|c|c|c|}
\hline \multirow{2}{*}{ Helminths species } & \multicolumn{3}{|c|}{ Age } & \multicolumn{2}{|c|}{ Sex } & \multirow{2}{*}{ Total $(n, \%)$} \\
\hline & $7-10(n, \%)$ & $11-14(n, \%)$ & $15-18(n, \%)$ & Male $(n, \%)$ & Female $(n, \%)$ & \\
\hline A. lumbricoides & $23(5.45)$ & $46(10.9)$ & $20(4.7)$ & $43(10)$ & $46(10.9)$ & $89(21.1)$ \\
\hline Hookworm spp & $40(9.47)$ & $83(19.66)$ & $25(5.9)$ & $79(18.7)$ & $69(16.4)$ & $148(35.1)$ \\
\hline S. mansoni & $16(3.79)$ & $33(7.8)$ & $10(2.36)$ & $35(8.3)$ & $24(5.7)$ & $59(13.9)$ \\
\hline Total & $79(18.72)$ & $162(38.38)$ & $55(13.03)$ & $157(37.2)$ & $139(32.9)$ & $296(70.1)$ \\
\hline
\end{tabular}

TABLE 3: Arithmetic mean of fecal egg count at baseline, after single-dose and redosing treatment of mebendazole and praziquantel among infected children in northwest Ethiopia, 2018.

\begin{tabular}{|c|c|c|c|c|c|}
\hline \multirow[b]{2}{*}{ Helminth species } & \multicolumn{5}{|c|}{ Mean egg count per gram of stool } \\
\hline & Baseline & $\begin{array}{l}\text { After single dose of } \\
\qquad \operatorname{MEB}(N)\end{array}$ & $\begin{array}{l}\text { After redosing of } \\
\operatorname{MEB}(N)\end{array}$ & $\begin{array}{c}\text { After single dose of } \\
\text { PZQ }(N)\end{array}$ & $\begin{array}{c}\text { After redosing of } \\
\text { PZQ }(N)\end{array}$ \\
\hline A. lumbricoides & 129 & 79 & 0 & NA & NA \\
\hline Hookworms & 281 & 215 & 39 & NA & NA \\
\hline S. mansoni & 42 & NA & NA & 24 & 0 \\
\hline
\end{tabular}

${ }^{*} \mathrm{NA}=$ not applicable; $\mathrm{MEB}=$ mebendazole; $\mathrm{PZQ}=$ praziquantel.

mansoni in its single dose and redosing after two weeks was $91.5 \%$ and $100 \%$, respectively (Table 4 ).

3.4. Egg Reduction Rate of Mebendazole and Praziquantel. The ERR of single-dose mebendazole against hookworm and $A$. lumbricoides infection was $53.1 \%$ and $76.4 \%$, respectively. The ERR of mebendazole after the redosing regimen was $100 \%$ and $98.6 \%$ to the respective A. lumbricoides and hookworm infections. The ERR of praziquantel against $S$. mansoni in its single dose and redosing regimen was $95.1 \%$ and $100 \%$, respectively (Table 5 ).

\section{Discussion}

Periodic evaluation of anthelminthic drug efficacy is ideal to overcome the burden of helminthic infections. In the present study, the prevalence of intestinal helminths at 95\% CI was $51.2 \%(46.4-55.9 \%)$. This result is lower than the previous studies conducted in rural Bahir Dar (58\%) [16] and Zarima town $82.4 \%$ [17]. This result is also comparable with the previous (54.9\%) prevalence report in Durbetie town [18] but higher than a report in Libo-Kemkem (27.9\%) [19] and
(16.7\%) in Gondar town [20]. The difference could be justified as the difference in the proper implementation of pure water, sanitation, and hygiene (WASH) and open defecation practices.

The prevalence of $A$. lumbricoides in the present study was $21.1 \%$ which is in line with a study done in Zarima town (22\%) [17]. However, the present finding is higher than reports in rural Bahir Dar (1.7\%) [16], Durbetie town (13.9\%) [18], and Libo-Kemkem (9\%) in Gondar town [20]. This could be justified as the variation in sanitation and proper utilization of latrine and endemicity of the parasite. A. lumbricoides is prevalent in areas with high altitudes.

In the present study, the prevalence of hookworm was $35.1 \%$. This finding is higher than the previous studies conducted in Zarima town (19\%) [17] and Libo-Kemkem $(8.2 \%)$ [19], but lower than in rural Bahir Dar (41.3\%) [16], Durbetie town (46.9\%) [18], and Ethiopia (7.6\%) [21]. This prevalence difference might be due to the difference in open defecation practices and shoe-wearing habits among children, the soil type, atmospheric moisture, and land surface temperature that are determinants of hookworm transmission [4]. 
TABLE 4: Cure rate of mebendazole and praziquantel among helminth-infected children of Andasa primary school in northwest Ethiopia, 2018.

\begin{tabular}{|c|c|c|c|c|c|c|}
\hline \multirow{2}{*}{ Helminth spp } & \multirow{2}{*}{$\operatorname{NIC}(N)$} & \multirow{2}{*}{$\operatorname{NCCS}(N)$} & \multicolumn{2}{|c|}{ CR of MEB (500 mg) } & \multicolumn{2}{|c|}{ CR of PZQ $(40 \mathrm{mg} / \mathrm{kg})$} \\
\hline & & & Initial dose $(N, \%)$ & Redosing $(N, \%)$ & Initial dose $(N, \%)$ & Redosing $(N, \%)$ \\
\hline A. lumbricoides & 89 & 85 & $51(60)$ & $85(100)$ & NA & NA \\
\hline Hookworms & 148 & 145 & $47(32.4)$ & $132(91.0)$ & NA & NA \\
\hline S. mansoni & 59 & 59 & NA & NA & $54(91.5)$ & $59(100)$ \\
\hline
\end{tabular}

${ }^{*} \mathrm{NA}=$ not applicable; NIC = number of infected children; NCCS = number of children who completed the study; MEB = mebendazole; $\mathrm{PZQ}=$ praziquantel; $\mathrm{CR}=$ cure rate.

TABLE 5: Egg reduction rate of mebendazole and praziquantel among helminth children of Andasa primary school in Bahir Dar Zuria district, northwest Ethiopia, 2018.

\begin{tabular}{lccccc}
\hline \multirow{2}{*}{ Helminth spp } & \multirow{2}{*}{ NCCS } & \multicolumn{2}{c}{ ERR of single-dose MEB } & \multicolumn{2}{c}{ ERR of single-dose PZQ } \\
& & Initial dose & Redosing & \multicolumn{2}{c}{ Initial dose } \\
Redosing \\
\hline A. lumbricoides & 85 & $65(76.4)$ & $85(100)$ & NA & NA \\
Hookworm spp. & 145 & $77(53.1)$ & $143(98.6)$ & NA & NA \\
S. mansoni & 59 & NA & NA & $56(94.9)$ & $59(100)$ \\
\hline
\end{tabular}

${ }^{*} \mathrm{ERR}$ = egg reduction rate; NA = not applicable; NCCS = number of children who completed the study; MEB = mebendazole; $\mathrm{PZQ}=$ praziquantel.

In the present study, the prevalence of $S$. mansoni was $13.9 \%$ which is in line with previous prevalence reports in rural Bahir Dar (12.2\%) [16] and in Chuahit (11.2\%) [22], but lower than prevalence reports (74.9\%) in Wondo Genet [23], Wolaita zone (81.3\%) [24], Southern Tigray (73.9\%) [25], Sanja town (35\%) [26], and northwest Ethiopia (83.3\%) [27]. The difference might be justified as the variation in the availability of water bodies and water contact habits of children. Children might be infected by cercaria when they are swimming, bathing, and washing their clothes in the river which is already infested with cercaria.

In the current study, the CR and ERR of single-dose mebendazole against $A$. lumbricoides were $60 \%$ and $76.4 \%$, respectively. The current result is lower than previous study reports in northwest Ethiopia [28] and Zanzibar [29]. This could be justified as the prolonged administration of mebendazole and repeated exposure with the same type of drug in the same area can result in reduced efficacy of mebendazole.

In the present study, the CR and ERR of MEB against hookworm in single-dose treatment were $32.4 \%$ and 55.1\%, respectively. Similarly, lower CR and ERR are also obtained in northwest Ethiopia [28] and in Vietnam [30]. This might be partially explained by hookworm losing its sensitivity to MEB that was previously sensitive due to the presence of alleles coding for resistance in hookworm as a result of mutations.

In the current study, the CR and ERR of MEB against hookworm and A. lumbricoides in redosing single-dose treatment regimen were higher than that of the single-dose regimen. This finding is consistent with a study done in northwest Ethiopia [31], Cameroon [11], and Lao PDR [32]. Since MEB is not effective in killing the eggs of A. lumbricoides and larvae of hookworm which become an adult within a few days, redosing gives a chance to kill these parasites.

This could be justified as redosing of MEB increases the serum half-life of the drug and is able to kill the tissue stages of the parasite in the primary dose.
The CR and ERR of single-dose praziquantel were 91.5\% in the present study. This finding is comparable with a previous report [27], but higher than earlier reports [33]. The difference in the low CR and ERR might be justified as the prolonged administration of praziquantel in endemic areas.

In the present study, the CR and ERR of single-dose praziquantel treatment against $S$. mansoni were increased in the redosing regimen. This result is consistent with previous reports from northern Ethiopia [34], western Ethiopia [35], and Sudan [36]. However, the current result is higher than that of a study conducted in southern Ethiopia [23] and Niger [37]. The CR and ERR of praziquantel difference in different settings could be due to the difference in baseline infection intensity, duration of posttreatment to recheck, presence of immature stages of the parasite in the tissue, immune status, and brand of praziquantel used for treatment.

\section{Conclusions}

The single-dose regimen of mebendazole for the treatment of hookworm and A. lumbricoides infections showed poor cure and egg reduction rates, while redosing showed satisfactory. Single-dose and redosing regimens of praziquantel for treatment of S. mansoni showed satisfactory cure and egg reduction rates. Therefore, single-dose praziquantel can be used as a treatment of $S$. mansoni infection whereas single-dose mebendazole for the treatment of $A$. lumbricoides and hookworm infections should be replaced by a redosing regimen.
Abbreviations
STH: Soil-transmitted helminth
MEB: Mebendazole
PZQ: Praziquantel
ERR: Egg reduction rate
MDA: Mass drug administration. 


\section{Data Availability}

The data used to support the findings of the study are included within the article.

\section{Ethical Approval}

The study protocol was evaluated and approved by the Institutional Review Board (IRB) of the College of Medicine and Health Sciences, Bahir Dar University, and ethical approval was obtained (protocol no. 069/18-04). Official supporting letters were also obtained from Amhara Public Health Institute and Zonal health offices.

\section{Consent}

Prior to commencing the study, verbal assent was collected from each participant, and informed consent was obtained from the guardians. Participant confidentiality and any special data security requirements were maintained and assured.

\section{Conflicts of Interest}

The authors declared they have no conflicting interest.

\section{Authors' Contributions}

All authors made substantial contributions to conception and design, acquisition of data, or analysis and interpretation of data; took part in drafting the article or revising it critically for important intellectual content; agreed on the journal to which the article will be submitted; gave final approval of the version to be published; and agreed to be accountable for all aspects of the work.

\section{Acknowledgments}

The data collection of the research project was funded by Bahir Dar University.

\section{References}

[1] L. T. Tchuenté, "Control of soil-transmitted helminths in subSaharan Africa: diagnosis, drug efficacy concerns and challenges," Acta Tropica, vol. 120, pp. S4-S11, 2011.

[2] C. Uneke, "Soil transmitted helminth infections and schistosomiasis in school age children in sub-Saharan Africa: efficacy of chemotherapeutic intervention since World Health Assembly resolution," Tanzania Journal of Health Research, vol. 2010, pp. 86-99, 2001.

[3] N. Negussu, B. Mengistu, B. Kebede et al., "Ethiopia schistosomiasis and soil-transmitted helminthes control programme: progress and prospects," Ethiopian Medical Journal, vol. 55, p. $75,2017$.

[4] A. A. Bitew, B. Abera, W. Seyoum et al., "Soil-transmitted helminths and Schistosoma mansoni infections in Ethiopian Orthodox church students around Lake Tana, Northwest Ethiopia," PLoS ONE, vol. 11, no. 5, p. e0155915, 2016.

[5] J. Bethony, S. Brooker, M. Albonico et al., "Soil-transmitted helminth infections: ascariasis, trichuriasis, and hookworm," The Lancet, vol. 367, no. 9521, pp. 1521-1532, 2006.
[6] T. Leenstra, P. A. Phillips-Howard, S. K. Kariuki et al., "Permethrin-treated bed nets in the prevention of malaria and anemia in adolescent schoolgirls in western Kenya," American Journal of Tropical Medicine Hygiene, vol. 68, 4_supplement, pp. 86-93, 2003.

[7] B. T. Crookston, S. C. Alder, I. Boakye et al., "Exploring the relationship between chronic undernutrition and asymptomatic malaria in Ghanaian children," Malaria Journal, vol. 9, no. 1,2010

[8] A. G. P. Ross, P. B. Bartley, A. C. Sleigh et al., "Schistosomiasis," New England Journal of Medicine, vol. 346, no. 16, pp. 1212-1220, 2002.

[9] B. Gryseels, K. Polman, J. Clerinx, and L. Kestens, "Human schistosomiasis," Lancet, vol. 368, no. 9541, pp. 1106-1118, 2006.

[10] World Health Organization, "Preventive chemotherapy in human helminthiasis: coordinated use of anthelminthic drugs in control interventions, a manual for health professionals and programme managers," 2006, September 2018 http://apps .who.int/iris/handle/10665/43535.

[11] V. K. Payne, L. Megwi, M. Noubom et al., "Efficacy of single dose of $500 \mathrm{mg}$ mebendazole on geohelminths amongst school age children in Bafoussam, Cameroon," British Microbiology Research Journal, vol. 12, no. 2, pp. 1-9, 2016.

[12] S. Geerts and B. Gryseels, "Drug resistance in human helminths: current situation and lessons from livestock," Clinical Microbiology Reviews, vol. 13, no. 2, pp. 207-222, 2000.

[13] D. De Clercq, M. Sacko, J. Behnke, F. Gilbert, P. Dorny, and J. Vercruysse, "Failure of mebendazole in treatment of human hookworm infections in the southern region of Mali," American Journal Tropical Medicine Hygiene, vol. 57, no. 1, pp. 2530, 1997.

[14] A. Ismail, S. Botros, A. Metwally et al., "Resistance to praziquantel: direct evidence from Schistosoma mansoni isolated from Egyptian villagers," American Journal Tropical Medicine Hygiene, vol. 60, no. 6, pp. 932-935, 1999.

[15] World Health Organization, "Assessing the efficacy of anthelminthic drugs against schistosomiasis and soil-transmitted helminthiases," 2013, November 2018 https://www.who.int/ iris/handle/10665/79019.

[16] T. Hailu, B. Abera, W. Mulu, M. Alemu, E. Yizengaw, and A. Genanew, "Efficacy of single dose albendazole and praziquantel drugs among helminth-infected school children at rural Bahir Dar, northwest Ethiopia," Tropical Doctor, vol. 48, no. 4, pp. 270-272, 2018.

[17] A. Alemu, A. Atnafu, Z. Addis et al., "Soil transmitted helminths and Schistosoma mansoni infections among school children in Zarima town, northwest Ethiopia," BMC Infectious Diseases, vol. 11, no. 1, 2011.

[18] T. Alelign, A. Degarege, and B. Erko, "Soil-transmitted helminth infections and associated risk factors among schoolchildren in Durbete town," Northwestern Ethiopia. Journal of Parasitology Research, vol. 2015, 2015.

[19] T. Addisu and A. Asmamaw, "A survey of soil-transmitted helminths infections and schistosomiasis mansoni among school children in Libo-Kemkem district, northwest Ethiopia: cross sectional study," American Journal of Health Research, vol. 3, no. 2, pp. 57-62, 2015.

[20] A. Ayele, Y. Tegegne, A. Derso, T. Eshetu, and A. J. Zeleke, "Prevalence and associated factors of intestinal helminths among kindergarten children in Gondar town, northwest 
Ethiopia," Pediatric Health Med Ther, vol. Volume 12, pp. 3541, 2021.

[21] G. T. Leta, K. Mekete, Y. Wuletaw et al., "National mapping of soil-transmitted helminth and schistosome infections in Ethiopia," Parasites \& Vectors, vol. 13, no. 1, p. 437, 2020.

[22] A. Alemu, Y. Tegegne, D. Damte, and M. Melku, "Schistosoma mansoni and soil-transmitted helminths among preschoolaged children in Chuahit, Dembia district, northwest Ethiopia: prevalence, intensity of infection and associated risk factors," BMC Public Health, vol. 16, no. 1, 2016.

[23] B. Erko, A. Degarege, K. Tadesse, A. Mathiwos, and M. Legesse, "Efficacy and side effects of praziquantel in the treatment of Schistosomiasis mansoni in schoolchildren in Shesha Kekele Elementary School, Wondo Genet, southern Ethiopia," Asian Pacific Journal of Tropical Biomedicine, vol. 2, no. 3, pp. 235-239, 2012.

[24] B. Alemayehu and Z. Tomass, "Schistosoma mansoni infection prevalence and associated risk factors among schoolchildren in Demba Girara, Damot Woide District of Wolaita zone, southern Ethiopia," Asian Pacific Journal of Tropical Medicine, vol. 8, no. 6, pp. 457-463, 2015.

[25] N. Abebe, B. Erko, G. Medhin, and N. Berhe, "Clinico-epidemiological study of Schistosomiasis mansoni in Waja-Timuga, District of Alamata, northern Ethiopia," Parasites \& Vectors, vol. 7, no. 1, p. 158, 2014.

[26] E. Woldegerima, A. G. Bayih, Y. Tegegne, M. Aemero, and A. Zeleke, "Prevalence and reinfection rates of Schistosoma mansoni and praziquantel efficacy against the parasite among primary school children in Sanja Town, northwest Ethiopia," Journal of parasitology research, vol. 2019, Article ID 3697216, 24 pages, 2019.

[27] B. Levecke, J. Vlaminck, L. Andriamaro et al., "Evaluation of the therapeutic efficacy of praziquantel against schistosomes in seven countries with ongoing large-scale deworming programs," International Journal for Parasitology: Drugs and Drug Resistance, vol. 14, pp. 183-187, 2020.

[28] A. J. Zeleke, A. G. Bayih, S. Afework, and S. J. Gilleard, “Treatment efficacy and re-infection rates of soil-transmitted helminths following mebendazole treatment in schoolchildren, Northwest Ethiopia," Tropical Medicine and Health, vol. 48, no. 1, p. 90, 2020.

[29] M. Albonico, Q. Bickle, M. Ramsan, A. Montresor, L. Savioli, and M. Taylor, "Efficacy of mebendazole and levamisole alone or in combination against intestinal nematode infections after repeated targeted mebendazole treatment in Zanzibar," Bulletin of the World Health Organization, vol. 81, no. 5, pp. 343352, 2003.

[30] C. Flohr, L. N. Tuyen, S. Lewis et al., "Low efficacy of mebendazole against hookworm in Vietnam: two randomized controlled trials," American Journal of Tropical Medicine and Hygiene, vol. 76, no. 4, pp. 732-736, 2007.

[31] T. Eshetu, M. Aemero, and A. J. Zeleke, "Efficacy of a single dose versus a multiple dose regimen of Mebendazole against hookworm infections among school children: a randomized open-label trial," BMC Infectious Diseases, vol. 20, no. 1, p. 376,2020

[32] P. A. Soukhathammavong, S. Sayasone, K. Phongluxa et al., "Low efficacy of single-dose albendazole and mebendazole against hookworm and effect on concomitant helminth infection in Lao PDR," PLoS Neglected Tropical Diseases, vol. 6, no. 1, p. e1417, 2012.
[33] A. Tesfie, G. Getnet, A. Abere et al., "Praziquantel is an effective drug for the treatment of Schistosoma Mansoni infection among school-aged children in northwest Ethiopia," Tropical medicine and health, vol. 48, 2020.

[34] T. Dejenie, T. Asmelash, and M. Abdelkadir, "Efficacy of praziquantel in treating Schistosoma mansoni infected school children in Tumuga and Waja, north Ethiopia," Momona Ethiopian Journal of Science, vol. 2, pp. 3-11, 2010.

[35] S. H. L. Golassa and Z. Mekonnen, "Prevalence of Schistosoma mansoni and effectiveness of praziquantel in school children in Finchaa valley, Ethiopia," Journal of Parasitology and Vector Biology, vol. 4, pp. 25-30, 2012.

[36] M. Amin, M. Swar, M. Kardaman, D. Elhussein, G. Nouman, and A. Mahmoud, "Treatment of pre-school children under 6 years of age for schistosomiasis: safety, efficacy and acceptability of praziquantel," Sudan Journal of Medical Sciences, vol. 7, pp. 67-76, 2012.

[37] A. Garba, M. S. Lamine, N. Barkiré et al., "Efficacy and safety of two closely spaced doses of praziquantel against Schistosoma haematobium and S. mansoni and re-infection patterns in school-aged children in Niger," Acta Tropica, vol. 128, no. 2, pp. 334-344, 2013. 